\title{
Study of heterosis and combining ability for earliness and vegetative traits in Cucumber (Cucumis sativus L.)
}

\section{Rajni Tiwari* and Dinesh Kumar Singh}

Department of Vegetable Science, College of Agriculture, G. B. Pant University of Agriculture and Technology, Pantnagar, Udham Singh Nagar - 263145 (Uttarakhand) INDIA

*Corresponding author. E-mail: rajnit728@gmail.com

Received: October 13, 2015; Revised received: March 14, 2016; Accepted: June 4, 2016

Abstract: The present investigation was undertaken to investigate the extent of heterosis and combining ability on earliness, vegetative traits and yield of Cucumber. A field experiment was designed in line $x$ tester mating design with 11 parents (including 8 parthenocarpic lines namely Pant parthenocarpic cucumber-2, Pant parthenocarpic cucumber-3, Nun-3139, Nun-3121, Nun-3141, Infifnity, Isatis, Kian, and 3 monoecious testers namely PCUC-8, PCUC15, Pant Kheera -1 aalso known as PCUC-28\}) and their $24 F_{1}$ hybrids to work out the heterosis and combining ability for earliness and yield characters. All traits pertinent to earliness and yield showed significant values for heterosis and combining ability. Appreciable heterosis in desirable direction was found over better parent and check parent by the cross PCUCP-3 x PCUC-15 for earliness characters viz. days to first female flower $(-71.18 \&-70.31)$, days to first harvest $(-3.40 \&-22.01)$ whereas Cross Kian $x$ PCUC-15 showed maximum number of fruits per plant $(8.36$ \& $106.35)$ and fruit yield (86.34 \& 210.74 respectively). The crosses PCUCP-3 x PCUC-15 and Nun-3139 x PCUC-8 showed significant specific combining ability for earliness and yield characters. Cross Nun-3139 x PCUC-8 showed significant yield regarding heterosis and SCA. Regarding general combining ability for earliness parent Isatis and for yield characters parent Kian stood in top. Cross Nun-3139 x PCUC-8 showed Maximum specific combining ability for node number to first female flower (-0.98), number of fruits per plant (3.39) and fruit yield (220.57).

Keywords: Combining ability, Cucumber, Earliness, Heterosis, Vegetative growth

\section{INTRODUCTION}

Cucumber (Cucumis sativus L., $2 \mathrm{n}=2 \mathrm{x}=14$ ) is one of the most important member of the family cucurbitaceae having 118 genera and 825 species. It is a warm season fruit vegetable crop. As a vegetable crop, cucumber has great economic importance. The immature fruits of cucumber are used as salad and for making pickles, rayata and even brined on commercial scale. (Bairagi et al., 2013) They are mainly used as refreshing material due to their low energy content. The fruit and seed possess cooling properties. The fruit is also used as astringent and antipyretic. Fruits are also good for people suffering from constipation, jaundice and indigestion. The fruits are mainly consumed as salad, preparation of cosmetic items, soap and cream and in many other ways. (Dhiman and Prakash, 2005). In Africa, ripe raw cucumber fruits are used as a cure for spruce, a disease that causes flattening of the villi and inflammation of the lining of small intestine. In IndoChina, cooked immature fruits are used to treat dysentery in children. (Grubben and Denton, 2004).

India being the naive place of cucumber possesses a vast range of genetic diversity. Considerable heterosis is manifested in cucumber for various traits like earliness, number of fruits per plant, and high yield.
Heterosis breeding is one of the most efficient tools to exploit the genetic diversity. Heterosis has contributed significantly towards increased crop production and it has become the basis of multi-billion dollar agro business in the world (Phillips, 1999).

For development of promising $\mathrm{F}_{1}$ hybrids, the identification of genetically superior parents is an important factor. The lack of progress in increased fruit of cucumber might be partially due to the meagre breeding effort relative to other crop or lack of variability for yield (Wehner et al., 1989). Sex ratio is one of the factor that leads to low and later yield in cucumber. Yield and many factors that affect it are subject to considerable environmental influence. Line $\mathrm{x}$ Tester analysis is a method, which helps to evaluate inbred line performance in $F_{1}$ combination, using combining ability and heterosis. Combining ability analysis (Sprague and Tatum, 1942) is one of the powerful tools available which gives the estimates of combining ability effect and aids in selecting desirable parents and crosses for further exploiatation. (Kumar et al., 2011). In actual plant breeding, combining abilities have found their principle use in predicting the performance of parents and hybrid population, in the form of test crosses or poly crosses. Owing to the existence of large variability it was considered worthwhile to take up the present 
investigation, therefore an experiment on $24 \mathrm{~F}_{1}$ hybrids obtained by crossing 8 parthenocarpic lines with 3 monoecious testers of cucumber in line $\mathrm{x}$ tester design was studied to investigate the extent of heterosis and combining ability for earliness and vegetative characters.

\section{MATERIALS AND METHODS}

The present experiment was conducted at Vegetable Research Centre, G.B. Pant University of Agriculture and Technology, Pantnagar during March, 2012 to May, 2013. The experimental site was located at an altitude of 243.83 meters above the mean sea level. The experimental block represent the Tarai belt of Shiwalik ranges of the Himalayas and is located between the $29^{\circ} \mathrm{N}$ latitude and $79.3^{\circ} \mathrm{E}$ longitude. The experiment was designed in Line $\mathrm{x}$ Tester mating system with Randomized Block Design with three replications under polyhouse condition in an area of $250 \mathrm{~m}^{2}$. The spacing between rows was kept $0.6 \mathrm{~m}$ and within plants $0.45 \mathrm{~m}$ and each genotype consisted of five plants. The parental lines consist of 3 monoecious testers viz. PCUC-8, PCUC-15 and Pant Khira-1 (PCUC-28) and 8 parthenocarpic lines viz. PCUCP-2 (Pant Parthenocarpic Cucumber-2), PCUCP-3 (Pant Parthenocarpic Cucumber-3), Nun-3139, Nun-3121, Nun-3141, Infinity, Isatis and Kian. Eleven parental lines of cucumber were crossed in line $\mathrm{x}$ tester mating design during March, 2012 to June, 2012 under polyhouse condition. Then their twenty-four $F_{1}$ crosses along with parents were evaluated during January, 2013 to May, 2013 in polyhouse to check out heterosis and combining ability. All cultural operations were carried out and data for all quantitative characters were recorded.

Observations were recorded from five randomly selected plants on days to first female flower, node number to first female flower, plant height $(\mathrm{cm})$, number of fruits per plant and fruit yield ( $q / h a)$. Information about general combining ability and specific combining ability were obtained by subjecting the data to Line $\mathrm{x}$ Tester analysis as outlined by Kempthorne (1957). Combining ability analysis was carried out according to Griffing (1956b) method II model I. In this approach, using a suitable statistical model the component variances due to general and specific combining ability was estimated. Heterosis was calculated over better parent (BP) and standard parent (SP) for each character by using the formula given by Fonseca and Patterson (1968). Parent Pant Kheera-1 (PCUC-28) was taken as standard parent for standard heterosis

\section{RESULTS AND DISCUSSION}

There were significant differences among the parental lines with respect to different characters studied. The mean performance of 11 parents (including 8 parthenocarpic lines and 3 monoecious testers) along with $24 \mathrm{~F}_{1}$ hybrids is given in Table 1 . The estimates of general combining ability (gca) effects of 11 parents for all the 5 quantitative characters are presented in Table 2. It would be imperative to mention that for the characters days to first female flower, node number to first female flower, the negative gca and sca effects were considered to be desirable as it indicates earliness. In the present study good general combiner for different traits have been identified on the basis of estimates of gca. For the characters days to first female flower nine parents exhibited significant gca effects ranging from 0.81 (Nun-3141 and Isatis) to 2.39 (Nun-3139). Significant gca effects in negative direction were observed in Pant Parthenocarpic Cucumber-3 (-0.61), Nun-3121 (-0.61), Nun-3141 (-0.81), Infinity (-0.65), Isatis $(-0.81)$ and PCUC-15 (-0.71). All the parents showed significant gca effect out of which six were in negative direction for the trait node number to first female flower. Maximum negative significant gca was recorded in Isatis (-0.39) and Kian (-0.39). Regarding plant height the extent of gca effect ranged from -0.69 (Nun-3139) to 0.83 (Nun-3121) where all the parents showed effect in positive direction except Kian. Only three parents showed significant positive gca effects in aspect of number of fruits per plant. Highest value was observed in the parent Kian (1.57). Maximum desirable fruit yield was observed in the parent Kian (287.96) followed by Nun-3141(116.77).

The general combining ability is primarily the function of additive and additive $\mathrm{x}$ additive gene action. The additive parental effects are of more practical use to the breeders than non-allelic interaction, if these are exploited through conventional selection methods. GCA effects would be more stable as compared to SCA effects. In general, additive effects are mainly due to polygenes producing fixable effects and indicate the capacity of variety in relation to all other varieties, it was crossed with. High GCA effects of a parent is a function of breeding value and hence due to additive gene effect and/or additive $\mathrm{x}$ additive interaction effect which represents the fixable components of genetic variance. Apparently, parents with good gca effect may be presumed to possess more favourable genes for the concerned traits. The specific combining ability (sca) effects of crosses for all characters are presented in Table 3. Analysis of sca effects for the character days to first female flower revealed that out of 24 crosses, 9 exhibited significant sca effects, where 5 were with negative values. The highest negative effect were shown by the cross PCUCP-3 x PCUC-15 (-2.03) followed by PCUCP-2 x PCUC-8 (-1.82) \& Nun-3141 x PCUC-15 (-1.22). 18 crosses showed significant effects for node number to first female flower out of which 10 were in desirable negative direction. The range varied between -0.98 (Nun-3139 x PCUC-8) and 1.47 (Nun-3139 x Pant Kheera-1). The highest negative significant sca effect was recorded for cross Nun-3139 x PCUC-8 (-0.98) followed by PCUCP-3 x Pant Kheera-1 (-0.60). The highest effects were recorded by cross Isatis x Pant Kheera-1 (-4.48) followed by Nun-3121 x Pant Kheera-1 (-2.50) and PCUCP-2 x PCUC-8 (-2.00). 18 crosses expressed significant sca effects for plant height in which 8 were 
Table 1. Mean values of parents and their $F_{1}$ hybrids for different traits in cucumber.

\begin{tabular}{|c|c|c|c|c|c|c|}
\hline Genotypes & $\begin{array}{l}\text { Days to } \\
\text { first } \\
\text { female } \\
\text { flower }\end{array}$ & $\begin{array}{l}\text { Node no. } \\
\text { to first } \\
\text { female } \\
\text { flower }\end{array}$ & $\begin{array}{l}\text { Days } \\
\text { to first } \\
\text { fruit } \\
\text { harvest }\end{array}$ & $\begin{array}{l}\text { Plant } \\
\text { height } \\
\text { (m) }\end{array}$ & $\begin{array}{l}\text { Number } \\
\text { of } \\
\text { Fruits per } \\
\text { plant }\end{array}$ & $\begin{array}{l}\text { Fruit } \\
\text { yield } \\
\text { (q/ha) }\end{array}$ \\
\hline PCUCP- 2 x PCUC-8 & 19.80 & 3.00 & 59.00 & 3.55 & 7.80 & 774.78 \\
\hline PCUCP-2 x PCUC-15 & 22.40 & 3.00 & 58.97 & 3.02 & 8.00 & 612.72 \\
\hline PCUCP-2 x Pant kheera-1 & 22.20 & 3.00 & 59.03 & 3.02 & 8.00 & 725.20 \\
\hline PCUCP-3 3 PCUC-8 & 20.20 & 2.80 & 59.00 & 3.40 & 7.40 & 580.97 \\
\hline PCUCP-3 x PCUC-15 & 16.60 & 3.00 & 53.03 & 2.85 & 10.20 & 751.10 \\
\hline PCUCP-3 x Pant kheera-1 & 21.20 & 2.40 & 59.00 & 3.57 & 9.80 & 908.72 \\
\hline NUN-3139 x PCUC-8 & 22.60 & 1.80 & 59.03 & 3.32 & 13.80 & 943.50 \\
\hline NUN-3139 x PCUC-15 & 21.40 & 2.20 & 53.00 & 2.77 & 11.40 & 788.10 \\
\hline NUN-3139 x Pant kheera-1 & 23.00 & 4.60 & 59.00 & 2.90 & 7.40 & 532.43 \\
\hline NUN-3121 x PCUC-8 & 20.40 & 3.20 & 59.00 & 4.25 & 8.80 & 689.68 \\
\hline NUN-3121 x PCUC-15 & 18.60 & 2.00 & 53.00 & 3.85 & 8.00 & 577.20 \\
\hline NUN-3121 x Pant kheera-1 & 19.00 & 2.60 & 53.00 & 5.45 & 7.00 & 551.30 \\
\hline NUN-3141 x PCUC-8 & 19.80 & 2.20 & 59.00 & 3.55 & 11.60 & 973.10 \\
\hline NUN-3141 x PCUC-15 & 17.20 & 2.20 & 53.00 & 4.17 & 9.40 & 865.80 \\
\hline NUN-3141 x Pant kheera-1 & 20.40 & 2.20 & 59.00 & 3.70 & 8.80 & 876.90 \\
\hline Infifnity x PCUC-8 & 19.00 & 2.20 & 59.00 & 4.05 & 6.00 & 589.04 \\
\hline Infifnity x PCUC-15 & 19.90 & 1.80 & 53.00 & 3.70 & 11.40 & 1002.10 \\
\hline Infifnity $\mathrm{x}$ Pant kheera-1 & 19.00 & 2.40 & 59.00 & 4.00 & 11.80 & 922.04 \\
\hline Isatis x PCUC-8 & 19.80 & 1.80 & 59.00 & 5.12 & 6.00 & 529.84 \\
\hline Isatis $\mathrm{x}$ PCUC-15 & 18.60 & 2.20 & 59.00 & 3.75 & 9.00 & 754.80 \\
\hline Isatis x Pant kheera-1 & 19.00 & 2.20 & 53.03 & 3.40 & 10.20 & 745.18 \\
\hline Kian $x$ PCUC-8 & 19.20 & 2.00 & 59.00 & 3.65 & 9.80 & 973.10 \\
\hline Kian x PCUC-15 & 19.20 & 1.80 & 53.03 & 3.20 & 13.00 & 1231.36 \\
\hline Kian x Pant kheera-1 & 20.20 & 2.40 & 58.97 & 4.20 & 10.00 & 1024.90 \\
\hline Pant Parthenocarpic Cucumber-2 & 30.40 & 6.40 & 49.97 & 3.10 & 17.00 & 1176.00 \\
\hline Pant Parthenocarpic Cucumber-3 & 27.60 & 5.00 & 50.00 & 3.35 & 16.60 & 893.92 \\
\hline NUN-3139 & 30.80 & 5.80 & 46.00 & 2.70 & 15.20 & 862.10 \\
\hline NUN-3121 & 27.20 & 4.80 & 41.97 & 2.30 & 16.80 & 928.70 \\
\hline NUN-3141 & 28.80 & 4.00 & 46.00 & 2.10 & 14.20 & 821.40 \\
\hline Infifnity & 23.40 & 4.80 & 46.00 & 1.85 & 18.20 & 987.90 \\
\hline Isatis & 25.00 & 3.60 & 50.03 & 2.50 & 12.00 & 851.00 \\
\hline Kian & 22.00 & 3.60 & 50.00 & 1.70 & 12.00 & 660.82 \\
\hline PCUC-8 & 56.50 & 13.50 & 68.00 & 2.50 & 5.13 & 303.69 \\
\hline PCUC-15 & 57.60 & 11.20 & 68.00 & 3.89 & 5.40 & 329.67 \\
\hline Pant Kheera -1 (PCUC-28) & 55.90 & 9.40 & 68.00 & 3.97 & 6.30 & 396.27 \\
\hline Mean & 24.68 & 3.74 & 55.77 & 3.38 & 10.38 & 775.29 \\
\hline C.V. & 2.76 & 2.90 & 2.65 & 2.32 & 2.83 & 2.87 \\
\hline S.E.m \pm & 0.39 & 0.06 & 0.85 & 0.05 & 0.17 & 12.82 \\
\hline C.D. $(5 \%)$ & 1.11 & 0.18 & 2.41 & 0.13 & 0.48 & 36.19 \\
\hline
\end{tabular}

in positive direction. The highest effect was shown by Isatis x PCUC-8 (0.85) followed by Nun-3121 x Pant Kheera-1 (0.84) and Nun-3141 x PCUC-15 (0.64). In case of number of fruits per plant Nun-3139 x PCUC-8 (3.39) showed highest sca effect followed by Infinity $\mathrm{x}$ Pant Kheera-1 (2.30). Nun-3121 x PCUC-8 (2.12) showed third highest effect. For fruit yield all the crosses showed significant sca effect except 3 crosses. The maximum desirable fruit yield was shown by Nun-3139 x PCUC-8 (220.57) followed by PCUCP-3 $x$ Pant Kheera-1 (164.45) and Infinity x PCUC-15 (129.97). The results are in conformity with the findings of Munshi et al. (2006), Yudhvir and Sharma
(2006) and Sarkar and Sirohi (2005).

Specific combining ability effect (SCA) which represents the predominance of non-additive gene action is a major component that may be utilized in heterosis breeding. The significance of SCA effect elucidates the presence of genetic diversity among parents tested and illustrates the contribution of dominance and/or epistatic effect which represent the non fixable components of the genetic variation related to heterosis. The crosses showing sca effects involving parent with good gca could be exploited as $F_{1}$ hybrid breeding. Maximum $F_{1}$ hybrids exhibiting significant sca effects, showed high amount of heterosis 
Table 2. Estimates of general combining ability effects of parents for different traits in cucumber.

\begin{tabular}{|c|c|c|c|c|c|c|}
\hline Genotypes & $\begin{array}{l}\text { Days } \\
\text { to first } \\
\text { female } \\
\text { flower }\end{array}$ & $\begin{array}{l}\text { Node } \\
\text { number to } \\
\text { first } \\
\text { female } \\
\text { flower } \\
\end{array}$ & $\begin{array}{l}\text { Days to } \\
\text { first } \\
\text { harvest }\end{array}$ & $\begin{array}{l}\text { Plant } \\
\text { Height } \\
(\mathrm{m})\end{array}$ & $\begin{array}{l}\text { Number of } \\
\text { fruits per } \\
\text { Plant }\end{array}$ & $\begin{array}{l}\text { Fruit yield } \\
\text { (q/ha) }\end{array}$ \\
\hline Pant Parthenocarpic Cucumber-2 & $1.52 * *$ & $0.54 * *$ & $2.00 * *$ & $-0.49 * *$ & $-1.42 * *$ & $-84.26 * *$ \\
\hline Pant Parthenocarpic Cucumber -3 & $-0.61 * *$ & $0.27 * *$ & 0.01 & $-0.41 * *$ & $-0.23 *$ & $-41.57 * *$ \\
\hline NUN-3139 & $2.39 * *$ & $0.41 * *$ & 0.01 & $-0.69 * *$ & $1.51 * *$ & $-33.82 * *$ \\
\hline NUN-3121 & $-0.61 * *$ & $0.14 * *$ & $-2.00 * *$ & $0.83 * *$ & $-1.42 * *$ & $-182.43 * *$ \\
\hline NUN-3141 & $-0.81 * *$ & $-0.26 * *$ & 0.00 & $0.12 * *$ & $0.57 * *$ & $116.77 * *$ \\
\hline Infifnity & $-0.65 * *$ & $-0.33 * *$ & 0.00 & $0.23 * *$ & $0.38 * *$ & $49.23 * *$ \\
\hline Isatis & $-0.81 * *$ & $-0.39 * *$ & 0.01 & $0.41 * *$ & $-0.96 * *$ & $-111.89 * *$ \\
\hline Kian & -0.41 & $-0.39 * *$ & 0.00 & 0.00 & $1.57 * *$ & $287.96 * *$ \\
\hline PCUC-8 & 0.15 & $-0.08 * *$ & $2.00 * *$ & $0.18 * *$ & $-0.46 * *$ & $-31.74 * *$ \\
\hline PCUC-15 & $-0.71 * *$ & $-0.18 * *$ & $-2.50 * *$ & $-0.27 * *$ & $0.69 * *$ & $34.40 * *$ \\
\hline Pant kheera-1 & $0.55 * *$ & $0.27 * *$ & 0.50 & $0.09 * *$ & $-0.23 * *$ & -2.66 \\
\hline $\mathrm{CD}$ at $5 \% \mathrm{GCA}($ Line $)$ & 0.46 & 0.07 & 0.99 & 0.05 & 0.20 & 14.90 \\
\hline $\mathrm{CD}$ at $5 \% \mathrm{GCA}($ Tester $)$ & 0.28 & 0.04 & 0.61 & 0.03 & 0.12 & 9.13 \\
\hline
\end{tabular}

*Significant at $5 \%$

**Significant at $1 \%$

Table 3. Estimate of SCA effects for different traits in cucumber.

\begin{tabular}{|c|c|c|c|c|c|c|}
\hline Genotypes & $\begin{array}{c}\text { Days } \\
\text { to first } \\
\text { female } \\
\text { flower } \\
\end{array}$ & $\begin{array}{l}\text { Node num- } \\
\text { ber to first } \\
\text { female } \\
\text { flower }\end{array}$ & $\begin{array}{c}\text { Days to } \\
\text { first } \\
\text { harvest }\end{array}$ & $\begin{array}{c}\text { Plant } \\
\text { Height (m) }\end{array}$ & $\begin{array}{l}\text { Number of } \\
\text { fruits per } \\
\text { Plant }\end{array}$ & $\begin{array}{l}\text { Fruit yield } \\
\qquad(q / h a)\end{array}$ \\
\hline PCUCP-2 x PCUC-8 & $-1.82 * *$ & 0.08 & $-2.00 *$ & $0.18 * *$ & 0.32 & $102.29 * *$ \\
\hline PCUCP-2 x PCUC-15 & $1.64 * *$ & $0.18 * *$ & $2.47 * *$ & $0.10 *$ & $-0.63 * *$ & $-125.92 * *$ \\
\hline PCUCP-2 x Pant Kheera-1 & 0.18 & $-0.26 * *$ & -0.47 & $-0.27 * *$ & 0.30 & 23.63 \\
\hline PCUCP-3 x PCUC-8 & 0.71 & $0.15^{*}$ & -0.01 & -0.05 & $-1.27 * *$ & $-134.22 * *$ \\
\hline PCUCP-3 x PCUC-15 & $-2.03 * *$ & $0.45 * *$ & -1.48 & $-0.15 * *$ & $0.38 *$ & $-30.23 *$ \\
\hline PCUCP-3 x Pant Kheera-1 & $1.31 * *$ & $-0.60 * *$ & 1.49 & $0.20 * *$ & $0.90 * *$ & $164.45^{* *}$ \\
\hline NUN-3139 x PCUC-8 & 0.11 & $-0.98 * *$ & 0.02 & $0.15 * *$ & $3.39 * *$ & $220.57 * *$ \\
\hline NUN-3139 x PCUC-15 & -0.23 & $-0.49 * *$ & -1.51 & 0.04 & -0.16 & -0.98 \\
\hline NUN-3139 x Pant Kheera-1 & 0.11 & $1.47 * *$ & 1.49 & $-0.19 * *$ & $-3.23 * *$ & $-219.59 * *$ \\
\hline NUN-3121 x PCUC-8 & $0.91 *$ & $0.68 * *$ & $2.00 *$ & $-0.44 * *$ & $1.33 * *$ & $115.37 * *$ \\
\hline NUN-3121 x PCUC-15 & -0.03 & $-0.41 * *$ & 0.50 & $-0.40 * *$ & $-0.62 * *$ & $-63.26 * *$ \\
\hline NUN-3121 x Pant Kheera-1 & $-0.89 *$ & $-0.27 * *$ & $-2.50 * *$ & $0.84 * *$ & $-0.70 * *$ & $-52.10 * *$ \\
\hline NUN-3141 x PCUC-8 & 0.51 & 0.08 & 0.00 & $-0.43 * *$ & $2.12 * *$ & $99.58 * *$ \\
\hline NUN-3141 x PCUC-15 & $-1.22 * *$ & $0.18 * *$ & -1.50 & $0.64 * *$ & $-1.22 * *$ & $-73.87 * *$ \\
\hline NUN-3141 x Pant Kheera-1 & 0.71 & $-0.27 * *$ & 1.50 & $-0.20 * *$ & $-0.90 * *$ & -25.71 \\
\hline Infifnity x PCUC-8 & -0.45 & $0.15 *$ & 0.00 & -0.04 & $-3.28 * *$ & $-216.94 * *$ \\
\hline Infifnity x PCUC-15 & $1.31 * *$ & $-0.15^{*}$ & -1.50 & 0.05 & $0.98 * *$ & $129.97 * *$ \\
\hline Infifnity x Pant Kheera-1 & $-0.86^{*}$ & 0.00 & 1.50 & -0.01 & $2.30 * *$ & $86.97 * *$ \\
\hline Isatis $\mathrm{x}$ PCUC- 8 & 0.51 & $-0.18 * *$ & -0.01 & $0.85^{* *}$ & $-1.94 * *$ & $-115.02 * *$ \\
\hline Isatis x PCUC-15 & 0.17 & $0.32 * *$ & $4.49 * *$ & -0.07 & -0.09 & $43.79 * *$ \\
\hline Isatis x Pant Kheera-1 & -0.69 & $-0.13 *$ & $-4.48 * *$ & $-0.79 * *$ & $2.03 * *$ & $71.23 * *$ \\
\hline Kian x PCUC-8 & -0.49 & 0.01 & 0.00 & $-0.21 * *$ & $-0.68 * *$ & $-71.61 * *$ \\
\hline Kian x PCUC-15 & 0.37 & -0.08 & -1.47 & $-0.21 * *$ & $1.38 * *$ & $120.51 * *$ \\
\hline Kian x Pant Kheera-1 & 0.12 & 0.07 & 1.47 & $0.42 * *$ & $-0.70 * *$ & $-48.89 * *$ \\
\hline $\mathrm{CD}$ at $5 \%$ & 0.79 & 0.13 & 1.72 & 0.09 & 0.34 & 25.81 \\
\hline
\end{tabular}

*Significant at $5 \% ; * *$ Significant at $1 \%$

for various characters. Dogra and Kanwar (2011) recorded high SCA estimates for yield and number of fruits. Mule et al. (2012) reported ratio of genetic variance was less than unity, which indicated the predominance of non-additive gene action in the entire traits. In the present study, the extent of heterosis was studied in $24 \mathrm{~F}_{1}$ hybrids of cucumber developed by 11 parents in line $\mathrm{x}$ tester design under protected condition. There was a wide variation in magnitude and direction of heterosis for all characters (Table 4). For the development of early fruiting genotypes, negative heterosis is desirable for days to first female flower, node number to first female flower and days taken to 1 st fruit harvesting. The magnitude of heterobeltiosis for Days to first female flower ranged from $-71.18 \%$ (PCUCP-3 x PCUC-15) to $-60.00 \%$ (Nun-3139 x PCUC-8).

The maximum heterobeltiosis was found in PCUCP-3 
Table 4. Heterosis in cucumber for earliness and vegetative characters.

\begin{tabular}{|c|c|c|c|c|c|c|c|c|c|c|c|c|}
\hline \multirow[t]{2}{*}{ Genotypes } & \multicolumn{2}{|c|}{$\begin{array}{c}\text { Days to first female } \\
\text { flower }\end{array}$} & \multicolumn{2}{|c|}{$\begin{array}{c}\text { Node number of } \\
\text { first female flower }\end{array}$} & \multicolumn{2}{|c|}{$\begin{array}{c}\text { Days to first } \\
\text { harvest }\end{array}$} & \multicolumn{2}{|c|}{ Plant height (m) } & \multicolumn{2}{|c|}{$\begin{array}{c}\text { Number of fruits per } \\
\text { plant }\end{array}$} & \multicolumn{2}{|c|}{ Fruit yield (q/ha) } \\
\hline & BP & $\mathrm{CP}$ & BP & $\mathrm{CP}$ & BP & $\mathbf{C P}$ & BP & $\mathbf{C P}$ & BP & $\mathrm{CP}$ & BP & $\mathbf{C P}$ \\
\hline $\begin{array}{l}\text { PCUCP-2 x PCUC } \\
-8\end{array}$ & $-64.96 *$ & $-64.58 * *$ & $-77.78 * *$ & $-68.09 * *$ & 3.41 & $-13.24 * *$ & $14.52 * *$ & $-10.58 * *$ & $-54.11 * *$ & $23.81 * *$ & $-34.12 * *$ & $95.52 * *$ \\
\hline $\begin{array}{l}\text { PCUCP-2 x PCUC } \\
-15\end{array}$ & $-61.11 * *$ & $-59.93 * *$ & $-73.22 * *$ & $-68.09 * *$ & 3.41 & $-13.28 * *$ & $-22.37 * *$ & $-23.93^{* *}$ & $-52.93 * *$ & $26.98 * *$ & $-47.90 * *$ & $54.62 * *$ \\
\hline $\begin{array}{l}\text { PCUCP-2 x Pant } \\
\text { Kheera-1 }\end{array}$ & $-60.29 * *$ & $-60.29 * *$ & $-68.05^{* *}$ & $-68.05^{* *}$ & 3.41 & $-13.19 * *$ & $-24.01^{* *}$ & $-24.01 * *$ & $-52.91 * *$ & $27.04 * *$ & $-38.33^{* *}$ & $83.01 * *$ \\
\hline $\begin{array}{l}\text { PCUCP-3 x PCUC } \\
-8\end{array}$ & $-64.25 * *$ & $-63.87 * *$ & $-79.26^{* *}$ & $-70.21 * *$ & -3.41 & $-13.24 * *$ & 1.39 & $-14.36^{* *}$ & $-55.40 * *$ & $17.51 * *$ & $-35.01 * *$ & $46.61 * *$ \\
\hline $\begin{array}{l}\text { PCUCP-3 x PCUC } \\
-15\end{array}$ & C-71.18 & $-70.31 * *$ & $-73.22 * *$ & $-68.09 * *$ & -3.40 & $-22.01 * *$ & $-26.74 * *$ & $-28.21 * *$ & $-38.55^{* *}$ & $61.90 * *$ & $-15.98 * *$ & $89.54 * *$ \\
\hline $\begin{array}{l}\text { PCUCP-3 x Pant } \\
\text { Kheera-1 }\end{array}$ & $-62.08 * *$ & $-62.08 * *$ & $-74.47 * *$ & $-74.47 * *$ & 3.41 & $-13.24 * *$ & $-10.08^{* *}$ & $-10.08 * *$ & $-40.98 * *$ & $55.50 * *$ & 1.66 & $129.32 * *$ \\
\hline $\begin{array}{l}\text { NUN-3139 x } \\
\text { PCUC-8 }\end{array}$ & $-60.00 * *$ & $-59.57 * *$ & $-86.67 * *$ & $-80.85^{* *}$ & 3.41 & $-13.19 * *$ & $23.09 * *$ & $-16.29 * *$ & $-9.21 * *$ & $119.05^{* *}$ & $9.44 * *$ & $138.10 * *$ \\
\hline $\begin{array}{l}\text { NUN-3139 x } \\
\text { PCUC-15 }\end{array}$ & $-62.85^{* *}$ & $-61.72 * *$ & $-80.39 * *$ & $-76.63 * *$ & -3.41 & $-22.06 * *$ & $-28.88 * *$ & $-30.31 * *$ & $-25.02 * *$ & $80.90 * *$ & $-8.58^{* *}$ & $98.88^{* *}$ \\
\hline $\begin{array}{l}\text { NUN-3139 x Pant } \\
\text { Kheera-1 }\end{array}$ & $-58.86^{* *}$ & $-58.86^{* *}$ & $-51.06^{* *}$ & $-51.06 * *$ & -3.41 & $-13.24 * *$ & $-26.95 * *$ & $-26.95 * *$ & $-51.32 * *$ & $17.46^{* *}$ & $-38.24 * *$ & $34.36 * *$ \\
\hline $\begin{array}{l}\text { NUN-3121 x } \\
\text { PCUC-8 }\end{array}$ & $-63.89 * *$ & $-63.51 * *$ & $-76.30 * *$ & $-65.96 * *$ & 3.41 & $-13.24 * *$ & $70.00 * *$ & $7.05^{* *}$ & $-47.62^{* *}$ & $39.68 * *$ & $-25.74 * *$ & $74.05 * *$ \\
\hline $\begin{array}{l}\text { NUN-3121 x } \\
\text { PCUC-15 }\end{array}$ & $-67.71 * *$ & $-66.73 * *$ & $-82.12 * *$ & $-78.69 * *$ & -3.41 & $-22.06 * *$ & -1.03 & -3.02 & $-52.38 * *$ & $26.98 * *$ & -37.8 & $45.66^{* *}$ \\
\hline $\begin{array}{l}\text { NUN-3121 x Pant } \\
\text { Kheera-1 }\end{array}$ & $-66.01 * *$ & $-66.01 * *$ & $-72.34 * *$ & $-72.34 * *$ & -3.40 & $-22.06^{* *}$ & $37.28 * *$ & $37.28 * *$ & $-58.33 * *$ & $11.11^{* *}$ & $-40.64 * *$ & $39.12 * *$ \\
\hline $\begin{array}{l}\text { NUN-3141 x } \\
\text { PCUC-8 }\end{array}$ & $-64.96 * *$ & $-64.58 * *$ & $-83.70 * *$ & $-76.60 * *$ & 3.41 & $-13.24 * *$ & $42.00 * *$ & $-10.58^{* *}$ & $-18.31 * *$ & $84.07 * *$ & $18.47 * *$ & $145.57 * *$ \\
\hline $\begin{array}{l}\text { NUN-3141 x } \\
\text { PCUC-15 }\end{array}$ & $-70.13 * *$ & $-69.23 * *$ & $-80.36^{* *}$ & $-76.60 * *$ & -3.41 & $-22.06 * *$ & $7.28 * *$ & $5.12 * *$ & $-33.79 * *$ & $49.21 * *$ & $5.40^{*}$ & $118.49 * *$ \\
\hline $\begin{array}{l}\text { NUN-3141 x Pant } \\
\text { Kheera-1 }\end{array}$ & $-63.51 * *$ & $-63.51 * *$ & $-76.63 * *$ & $-76.63 * *$ & 3.41 & $-13.24 * *$ & $-6.80 * *$ & $-6.80 * *$ & $-38.01 * *$ & $39.68 * *$ & $6.76^{* *}$ & $121.29 * *$ \\
\hline $\begin{array}{l}\text { Infifnity x PCUC- } \\
8\end{array}$ & $-66.37 * *$ & $-66.01 * *$ & $-83.70 * *$ & $-76.60 * *$ & 3.41 & $-13.24 * *$ & $62.13 * *$ & 2.10 & $-67.03 * *$ & -4.76 & $-40.37 * *$ & $48.65^{* *}$ \\
\hline $\begin{array}{l}\text { Infifnity x PCUC- } \\
15\end{array}$ & $-65.45^{* *}$ & $-64.40 * *$ & $-83.93 * *$ & $-80.85^{* *}$ & -3.41 & $-22.06^{* *}$ & -4.97 & $-6.88 * *$ & $-37.36 * *$ & $80.95 * *$ & 1.44 & $152.88 * *$ \\
\hline $\begin{array}{l}\text { Infifnity x Pant } \\
\text { Kheera-1 }\end{array}$ & $-66.02 * *$ & $-66.02 * *$ & $-74.47 * *$ & $-74.47 * *$ & 3.41 & $-13.24 * *$ & 0.84 & 0.84 & $-35.16 * *$ & $87.30 * *$ & $-6.67 * *$ & $132.68 * *$ \\
\hline Isatis $\mathrm{x}$ PCUC-8 & $-64.95 * *$ & $-64.58 * *$ & $-86.67 * *$ & $-80.85^{* *}$ & 3.41 & $-13.24 * *$ & 104.53 & $28.97 * *$ & $-49.96 * *$ & -4.71 & $-37.74 * *$ & $33.71 * *$ \\
\hline Isatis x PCUC-15 & $-67.71 * *$ & $-66.73 * *$ & $-80.36^{* *}$ & $-76.60 * *$ & 3.41 & $-13.24 * *$ & -3.51 & $-5.46^{* *}$ & $-24.98 * *$ & $42.86^{* * *}$ & $-11.30^{* *}$ & $90.48^{* *}$ \\
\hline $\begin{array}{l}\text { Isatis x Pant } \\
\text { Kheera-1 }\end{array}$ & $-66.01 * *$ & $-66.01 * *$ & $-76.60 * *$ & $-76.60 * *$ & -3.41 & $-22.01 * *$ & -14.36 & $-14.36^{* *}$ & $-14.95 * *$ & $61.96 * *$ & $-12.43 * *$ & $88.05 * *$ \\
\hline Kian x PCUC-8 & $-66.02 * *$ & $-65.66^{* *}$ & $-85.19 * *$ & $-78.72 * *$ & 3.41 & $-13.24 * *$ & 46.00 & $-8.06 * *$ & $-18.34 * *$ & $55.50 * *$ & $47.26 * *$ & $145.57 * *$ \\
\hline Kian x PCUC-15 & $-66.66 * *$ & $-65.66^{* * *}$ & $-83.90 * *$ & $-80.82 * *$ & -3.40 & $-22.01 * *$ & -17.74 & $-19.40^{* *}$ & $8.36 * *$ & $106.35 * *$ & $86.34 * *$ & $210.74 * *$ \\
\hline $\begin{array}{l}\text { Kian x Pant } \\
\text { Kheera-1 }\end{array}$ & $-63.86 * *$ & $-63.86^{* *}$ & $-74.43 * *$ & $-74.43 * *$ & 3.41 & $-13.28 * *$ & 5.79 & $5.79 * *$ & $-16.64 * *$ & $58.73 * *$ & $55.09 * *$ & $158.64 * *$ \\
\hline
\end{tabular}

*Significant at 5\%; **Significant at $1 \%$

x PCUC-15 (-71.18\%) followed by Nun-3141 x PCUC $-15(-70.13 \%)$ and Nun-3121 x PCUC-15 (-67.71\%). The magnitude of standard heterosis for that trait ranged from $-70.31 \%$ (PCUCP-3 $x$ PCUC-15) to $-58.86 \%$ (Nun-3139 x Pant Kheera-1). The maximum standard heterosis was found for the cross PCUCP-3 $\mathrm{x}$ PCUC-15 (-71.18\%) followed by Nun-3121 x PCUC$15(-66.73 \%)$ and Isatis x PCUC-15 (-66.73\%). the magnitude of heterobeltiosis for node number to first female flower ranged from $-86.67 \%$ (Nun-3139 x PCUC-8) to $51.06 \%$ (Nun-3139 x PCUC-28). The maximum heterobeltiosis was found in Nun-3139 $\mathrm{x}$ PCUC-8 (-86.67\%), Isatis x PCUC-8 (-86.67\%) and Kian x PCUC-8 (-85.19\%). The magnitude of standard heterosis for that trait ranged from $-80.85 \%$ (Infinity $\mathrm{x}$ PCUC-15) to $-51.06 \%$ (Nun-3139 x Pant Kheera-1). For plant height the scale of heterobeltiosis ranged from $-1.03 \%$ (Nun-3121 x PCUC-15) to $104.53 \%$
(Isatis x PCUC-8). Fourteen crosses exhibited significant heterosis over better parent out of which seven crosses were in positive direction. The maximum heterobeltiosis was found in Nun-3121 x PCUC$8(70.00 \%)$ followed by Infinity x PCUC-8 $(62.13 \%)$ and Nun-3141 x PCUC-8 $(42.00 \%)$. The degree of standard heterosis for that trait ranged from $-3.02 \%$ (Nun-3121 x PCUC-15) to $37.28 \%$ (Nun-3121 x Pant Kheera-1). For standard parent 21 crosses showed significant heterosis in which 5 recorded positive heterosis over standard parent. The maximum standard heterosis was found for the cross Nun-3121 x Pant Kheera $-1(37.28 \%)$ followed by Isatis x PCUC-8 (28.97\%). Regarding the trait number of fruits per plant the level of heterobeltiosis ranged from $-67.03 \%$ (Infinity $\mathrm{x}$ PCUC-8) to $8.36 \%$ (Kian x PCUC-15). All the crosses were found to be negatively significant over the better parent expect cross Kian x PCUC-15 (8.36\%).The 
range of standard heterosis varied between $-4.76 \%$ (Infinity $\mathrm{x}$ PCUC-8) to $119.05 \%$ (Nun-3139 $\mathrm{x}$ PCUC-8). 22 crosses exhibited significant heterosis over standard parent in positive direction. The highest value of standard heterosis was estimated for $119.05 \%$ (Nun-3139 x PCUC-8) followed by Kian x PCUC-15 (106.35\%) and Infinity x Pant kheera-1 $(87.30 \%)$.

Regarding the trait fruit yield per plant the range of heterobeltiosis varied from - 47.90\% (PCUCP-2 x PCUC-15) to $86.34 \%$ (Kian x PCUC-15). 22 crosses showed significant heterobeltiosis out of which only 6 crosses were in positive direction. The maximum heterobeltiosis was recorded in cross Kian x PCUC-15 (86.34\%) followed by Kian x Pant Kheera-1 $(55.09 \%)$ and Kian x PCUC-8 (47.26\%). The range of standard heterosis for that trait ranged from $33.71 \%$ (Isatis $\mathrm{x}$ PCUC-8) to $210.74 \%$ (Kian x PCUC-15). All crosses exhibited significant heterosis over standard parent in positive direction. The highest value of standard heterosis found in crosses Kian x PCUC-15 (210.74\%) followed by Kian x Pant Kheera-1 (158.64\%) and Infinity x PCUC-15 (152.88\%). The extent of heterosis studied in $24 F_{1}$ hybrids of cucumber showed that there was a wide variation in magnitude and direction of heterosis for all characters (Table 4). Cizov (1945) found considerable heterosis under polyhouse for earliness.

The main objective of cucumber breeding is to increase fruit yield per plant. Pyzhenkov and Kosarreva (1981) concluded in their study that 7 hybrids showed desirable heterosis for total yield for both outdoor and greenhouse cultivation. Pandey et al. (2005) and Hanchinamani and Patil (2009) reported the maximum yield attributed to increase in average fruit weight and total number of fruits per plant. Bairagi et al. (2002), Kumbhar et al. (2005) and Munshi et al. (2005) found promising heterosis for fruit yield in cucumber. This indicated that there was a strong tendency of transmission of higher gain from the parents to the offspring

\section{Conclusion}

On the basis of above findings, it may be concluded that improvement in cucumber for yield and its contributing traits may be brought out through selection and hybridization. The gca and sca effects were highly significant for all the character studied which indicated that both additive and non additive gene actions were important in the inheritance of these characters. Therefore, in improvement of these traits, both selection and heterosis methods of breeding can be adopted. The crosses which showed high sca effects can be best utilized in heterosis breeding and the response to selection is expected to be the best in the crosses involving parents having high gca effects. The genotypes Isatis, Nun-3141, PCUC-15 and Kian can be used as suitable parent to develop commercial early hybrid having pro- fuse vegetative growth. The hybrids Nun-3139 x Pant Kheera -1, PCUCP-3 x Pant Kheera -1, PCUCP-2 x Pant Kheera-1and PCUCP-2 x PCUC-15 can commercially be released for earliness viz. days to first female flower, node number to first female flower and the hybrids Nun-3139 x PCUC-8, Infinity x PCUC-15, Infinity $\mathrm{x}$ Pant Kheera-1, Kian $\mathrm{x}$ PCUC-15 for vegetative traits viz. plant height and yield contributing characters like number of fruits per plant and fruit yield.

\section{ACKNOWLEDGEMENT}

Experimental facilities and assistance provided by Govind Ballabh Pant University of Agriculture and Technology, Pantnagar for conducting the research is duly acknowledged

\section{REFERENCES}

Bairagi, S.K., Singh, D.K. and Ram, H.H. (2002). Studies on heterosis for yield attribute in cucumber (Cucumis sativus L.). Veg. Sci., (29): 75-77.

Bairagi, S.K., Singh, D.K. and Ram, H.H. (2013). analysis of combining ability in cucumber (Cucumis sativus L.) through half diallel mating system. Annals of Horticulture., 6 (2): 308-314.

Cizov, S.I. (1945). Yield of hybrid cucumbers in glass house. Proc. Sci. Conf. Timirja Zeo. Agric. Acad., (1): 42-43.

Dhiman and Chander Prakash (2005). Correlation and path coefficient analysis in cucumber. Haryana J. Horti. Sci., 34 (1-2): 111-112.

Dogra, B.S. and Kanwar, M.S. (2011). Exploitation of combining ability in cucumber (Cucumis sativus L.) Res. $J$. of Agri. Sci., 2 (1): 55-59.

Fonseca and Patterson (1968). Hybrid vigour in a seven parent diallel crosses in common wheat (Triticum aestivum L.). Crop Sci., 8: 85-88.

Griffing, J.B. (1956b). Concept of general and specific combining ability in relation to diallel crossing system. Aus. J. Biol. Sci., 9: 463-493

Grubben, G.J.H. and Denton, O.A. (2004). Plant Resources of Tropical Africa 2. Vegetable. PROTA Foundation, Wageningen; Backhuys, Leiden; CTA, Wageningen. (4): 103-111.

Hanchinamani, C.N. and Patil, M.G. (2009). Heterosis in cucumber (Cucumis sativus L.) The Asian J. of Hort., 4 (1): 21-24.

Kempthorne, O. (1957). An introduction to genetic statistics, New York.

Kumar J., Munshi A.D., Kumar R., Sureja A.K. And Sharma R K. (2011). Combining ability and its relationship with gene action in slicing cucumber. Indian J. Hort. 68 (4): 507-511.

Kumbhar, H.C.; Dimbre, A.D. and Patil, H.E. (2005). Heterosis and combining ability studies in cucumber (Cucumis sativus L.). J. Maharastra Agr. Univ., 30 (3): 272-275.

Mule P.N., Khandelwal V, Lodam V.A., Shinde. D.A, Patil P.P. and Patil A.B. (2012). Heterosis and Combining Ability in Cucumber (Cucumis sativus L.) Madras Agric. J., 99 (7-9): 420-423.

Munshi, A.D., Kumar, R. and Panda, B. (2005). Heterosis for yield and its component in cucumber (Cucumis sativus 
L.). Veg. Sci., 32 (2): 133-135.

Munshi, A.D., Kumar, R. and Panda, B. (2006). Studies on genetic components of variation in cucumber (Cucumis sativus L.). Indian J. Hort., 63 (2): 213-214.

Pandey, P., Singh, B., Singh, M. and Rai, M. (2005). Hetersis in cucumber (Cucumis sativus L.). Veg. Sci., 32 (2): 143-145

Phillips, R.L. (1999). Research needs in heterosis. In: Coors, J. G. and Pandey, S. (eds.) Genetics and Exploitation of heterosis in crops. pp 501-507.

Pyzhenkov, V.I. and Kosarreva, G.A. (1981). Effect of heterosis in yield structure in cucumbers. Trudy po Prik- ladnoi Botanike Genetike Zselektsii. 65: 112-118.

Sarkar, M. and Sirohi P.S. (2005). Genetics of fruit character in cucumber (Cucumis sativus L.). Orissa J. Hort. 33:1-2.

Sprague G.F. and Tatum L.A. (1942). General versus specific combining ability in single cross of corn. J. Am. Soc. Agron., 34: 923-932.

Wehner, T.C., Lower, R.L., Staub, J.E. and Jolla, G.E. (1989). Convergent divergent selection for cucumber fruit yield. Hort. science, 24: 667-669.

Yudhvir, S. and Sharma, S. (2006). Combining ability through line $\mathrm{x}$ tester analysis in cucumber. Crop Res., 31: 110-15. 